\title{
Téoros
}

Revue de recherche en tourisme

\section{Terroirs, agrotourisme et marketing}

Le cas des fromages québécois

\section{Léonard Dumas, William Menvielle, Jocelyn D. Perreault et Denis Pettigrew}

Volume 25, numéro 1, printemps 2006

Entre la culture du goût et le goût de la culture

URI : https://id.erudit.org/iderudit/1071032ar

DOI : https://doi.org/10.7202/1071032ar

Aller au sommaire du numéro

Éditeur(s)

Université du Québec à Montréal

ISSN

0712-8657 (imprimé)

1923-2705 (numérique)

Découvrir la revue

Citer cet article

Dumas, L., Menvielle, W., Perreault, J. D. \& Pettigrew, D. (2006). Terroirs, agrotourisme et marketing : le cas des fromages québécois. Téoros, 25(1), 34-41. https://doi.org/10.7202/1071032ar d'utilisation que vous pouvez consulter en ligne.

https://apropos.erudit.org/fr/usagers/politique-dutilisation/ 


\section{Terroirs, agrotourisme et marketing Le cas des fromages québécois}

\section{Léonard Dumas, William Menvielle, Jocelyn D. Perreault et Denis Pettigrew ${ }^{1}$}

Outre les populaires cheddars et okas, fabriqués depuis plus de 110 ans, les fromages du Québec ont le vent en poupe! On produit maintenant dans toutes les régions du Québec une impressionnante gamme de fromages fins à base de lait de vache, de chèvre et de brebis. Les fromages produits au Québec représentent plus de $50 \%$ de la production totale canadienne. II y aurait au-delà de 200 sortes de fromages québécois. Si l'on en juge par les résultats d'une étude récente sur le sujet, l'agrotourisme est en plein essor et en voie de devenir une activité importante pour soutenir et diversifier le développement économique des régions du Québec (Marcotte et Bourdeau, 2004).

Malgré ce portrait idyllique, l'avenir des producteurs est loin d'être assuré. Le dossier des "appellations " est toujours d'actualité et il reste encore beaucoup à faire pour préserver ces produits de terroir d'usurpations éventuelles de leur dénomination et pour assurer efficacement leur mise en marché. Cet article propose une réflexion sur la question axée sur une perspective de marketing touristique et sur les interrelations entre les producteurs, les restaurateurs et les consommateurs.

Le Groupe de concertation sur l'agrotourisme au Québec a pour mission de promouvoir et de favoriser le développement de l'agrotourisme. Pour cet organisme, l'agrotourisme représente une activité touristique complémentaire de l'agriculture qui a lieu dans une exploitation agricole. II met des producteurs agricoles en relation avec des

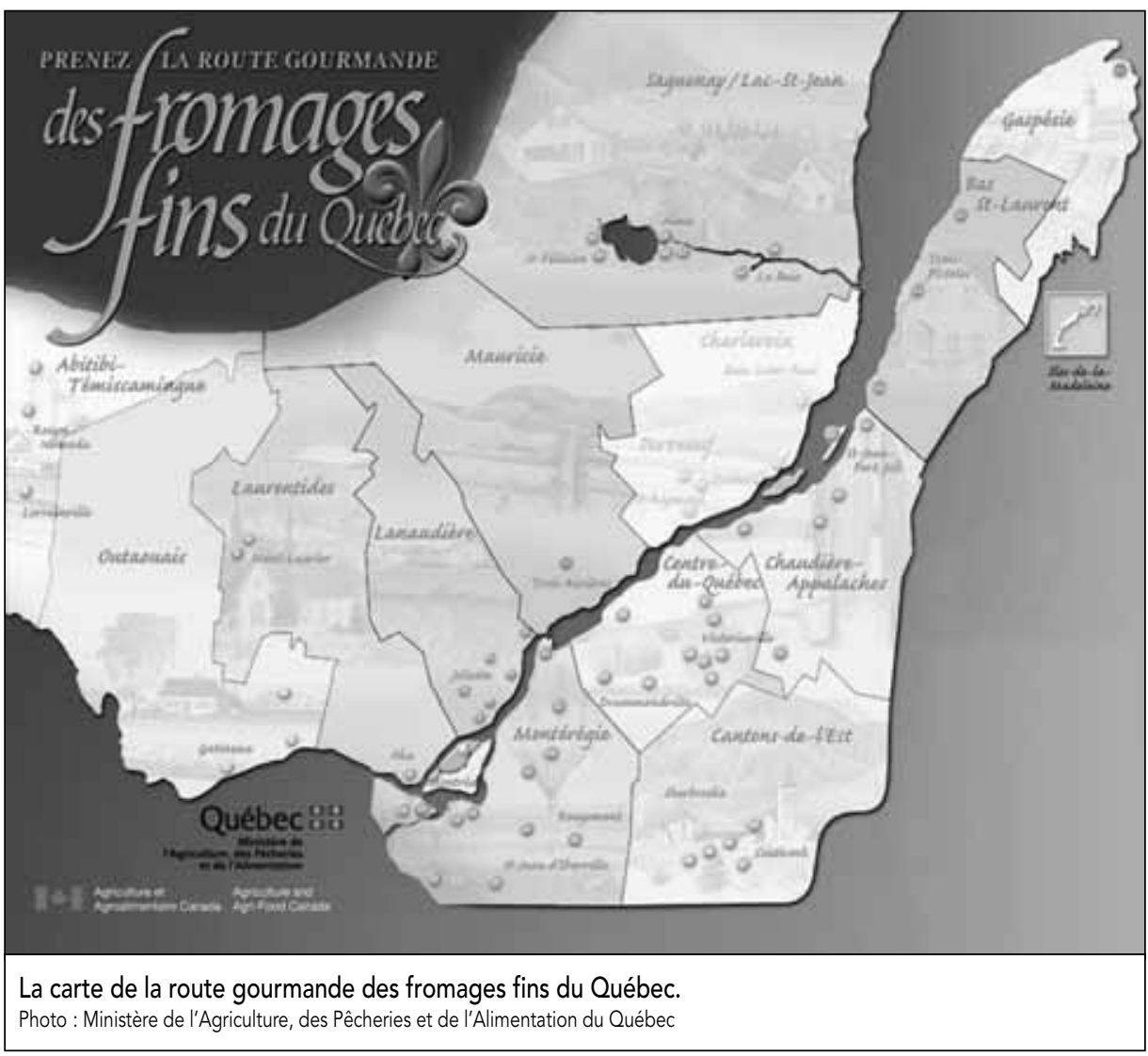

touristes ou des excursionnistes, leur donnant ainsi la possibilité de découvrir le milieu agricole, l'agriculture et sa production, par l'accueil et l'information que leur réserve leur hôte (MAPAQ, 2003).

L'offre agrotouristique se manifeste principalement par la mise en œuvre de visites ou d'activités d'animation à la ferme, par l'hébergement, la restauration où l'on porte l'accent sur la mise en valeur de produits de la ferme et de produits régionaux qui constituent les éléments principaux du menu ou, encore, par la promotion et la vente de produits agroalimentaires. C'est grâce aux particularités de son accueil et à la diffusion d'information à caractère agricole que l'agrotourisme se distingue des autres formes de tourisme. L'agrotourisme est appelé à jouer un rôle à multiples facettes. Sur le plan économique, il soutient et diversifie les revenus agricoles tout en facilitant la mise en marché de produits et services agricoles ou alimentaires; en outre, ces activités contribuent à valoriser la contribution de l'agriculture à l'économie régionale québécoise. Sur les plans social, éducatif et touristique, en plus de sensibiliser le public à l'agriculture et à l'usage du territoire agricole, il ajoute des expériences touristiques nouvelles et diversifiées. 
Par ailleurs, les faits saillants de l'étude de Marcotte et Bourdeau (2004) permettent de mieux cerner le phénomène de l'agrotourisme. On estime qu'il existe environ 600 entreprises qui œuvrent dans le domaine au Québec. De ce nombre, 136 producteurs ont affirmé accueillir plus de 1,2 million de visiteurs annuellement. Les revenus minimaux associés aux activités agrotouristiques représentent environ 100000 \$ par année. En moyenne, chacun des producteurs dépense annuellement 5000 \& en publicité. En outre, les producteurs constatent que plus ils consacrent de ressources aux activités agrotouristiques, plus le nombre de visiteurs augmente. Les activités agrotouristiques qu'ils organisent contribuent à la croissance de leur chiffre d'affaires. Finalement, les acteurs dans ce domaine ont choisi l'agrotourisme parce qu'ils aiment le public et qu'ils souhaitent leur faire connaître le milieu agricole (FSA, 2004).

Toutefois, toujours selon le Groupe de concertation sur l'agrotourisme au Québec, certaines conditions doivent être réunies afin que l'offre agrotouristique connaisse le succès. Notamment, celle-ci devrait s'intégrer à l'offre touristique du Québec, mettre en valeur la production agricole et ses dérivés, adopter une pratique d'animation et d'accueil dont la dimension "communication" est caractérisée par le "savoir-faire » et le goût de transmettre son expérience et, finalement, axer sa stratégie de marketing sur un agrotourisme authentique. Or, malgré les initiatives des producteurs, il y a péril en la demeure puisque, à ce jour, l'authenticité des produits ne saurait être garantie.

Appellation d'origine contrôlée, produit régional, produit du terroir, produit artisanal, produit gourmand, produit de niche... la route est longue. Comment les consommateurs et les intervenants du milieu peuventils s'y retrouver dans cette avalanche de dénominations? Pourtant, la croissance marquée des produits régionaux et des produits de niche, dont on évalue la demande potentielle à plus de un milliard de dollars, représente environ $4 \%$ de la demande alimentaire totale (Lessard, 2005). Voilà ce qui explique en partie l'intérêt manifesté à l'automne 2005 par les auteurs des 20 mémoires déposés à la Commission de l'Agriculture, des Pêcheries et de l'Alimentation en vue de l'adoption prochaine d'un projet de loi modifiant la loi sur les appellations réservées, sanctionnée en 1996.
Nous allons nous attarder à la définition classique d'un produit du «terroir » et expliquer brièvement les enjeux liés aux appellations réservées en relation avec l'agrotourisme. Dans une perspective marketing, nous présenterons ensuite les résultats d'une recherche exploratoire réalisée pour le compte du Laboratoire de recherche et d'intervention en gestion hôtelière et de restauration de l'Université du Québec à Trois-Rivières (UQTR) à l'été 2005, portant sur la perception des produits de terroir selon les trois points de vue suivants : producteurs - restaurateurs - consommateurs. Nous compléterons cet article en suggérant quelques avenues de recherches futures sur le sujet.

\section{Le terroir, un concept flou}

À ce jour, à peu près n'importe qui peut apposer la mention «terroir » sur n'importe quoi. Cet état de fait a d'ailleurs été rapporté à l'émission L'Épicerie à l'occasion d'un reportage en avril 2004 (Radio-Canada, 2004) où, dans un épisode de la désormais célèbre série télévisée Les Bougon, $\mathrm{M}^{\mathrm{me}}$ Bougon affirmait sans ambages son appréciation de la chose: "prendre une confiture commerciale, la mettre dans un joli contenant, prétendre qu'elle est maison et la vendre plus cher! ». Mais, trêve de plaisanteries, la question qui se pose consiste à savoir ce qui se cache sous le vocable «terroir».

Malgré les recherches entreprises sur le sujet, plusieurs individus affirment toujours que le mot «terroir » demeure flou. Tout est question de perspective. Les propos d'Anne Desjardins, présidente du groupe de travail du même nom sur les appellations réservées et les produits du terroir, reflètent le point de vue de nombreux intervenants, notamment les restaurateurs et certains producteurs: "Quand un produit va porter sur son étiquette le mot terroir, artisanal ou fermier, il faudrait que ce soit défini. Qu'est-ce que cela veut dire ? » (Radio-Canada, 2004)

Par ailleurs, un point semble faire consensus et gravite autour de l'interprétation d'un produit de terroir proposée par le sociologue Granovetter, qui affirme que le produit est en quelque sorte ancré, encastré sur un territoire situé dans une zone géographique donnée (Granovetter, 2000). Pour leur part, Rastoin et Vissac-Charles (1999) s'en tiennent à une définition du terroir intégrant le concept de l'entreprise de terroir qui tire sa spécificité de liens de forte intensité avec un terroir identifié par des caractéristiques physiques, géographiques et agroclimatiques, historiques et sociales, donc culturelles. Quant à Marchesnay (2002 : 2), il propose l'argumentaire suivant : «[...] pour que les produits développés spécifiquement sur un territoire accèdent à une compétence réellement distinctive liée au statut de terroir, il importe que la symbiose s'opère entre trois exigences: il faut une histoire, un sol, et une volonté entrepreneuriale collective». Dans le même ordre d'idées, Bérard et Marchesnay (2000) élargissent leur domaine de recherche aux caractéristiques ethnocentriques du terroir, de manière à y intégrer une forte dimension historique qui sert à expliquer et à interpréter le mode de constitution du terroir, en tant que source d'avantage concurrentiel reposant sur des ressources ou des compétences distinctives.

Selon les perspectives marketing et agrotouristique, l'origine des produits alimentaires représente, pour les entreprises du secteur agroalimentaire, un important critère de différenciation qui constitue le fondement d'une offre d'expériences touristiques nouvelles et diversifiées et susceptibles de conduire à la création de valeur. Les consommateurs accordent habituellement au produit de terroir des attributs tels que naturels, traditionnels, authentiques ou, encore, de meilleure qualité (Warnier, 1994 ; Cova et Cova, 2001; Camus, 2002). À la suite d'une recherche exploratoire qualitative puis quantitative, Aurier et ses collègues (2004) font ressortir trois facteurs sources caractéristiques du terroir : la référence à la géographie, au temps et à la culture, ainsi qu'à un savoirfaire. Soulignons que l'objectif de leur étude était de mieux comprendre le concept de terroir du point de vue du consommateur et d'identifier les sources perçues et les associations au terroir. Bien que les auteurs mettent en garde le lecteur de toute intention de généraliser les résultats, étant donné la faible taille de l'échantillon ( $n=53)$, il n'en demeure pas moins que cette étude amène un éclairage additionnel sur notre compréhension du terroir. Dans cette étude exploratoire, il est intéressant de constater la mise en évidence de trois facteurs qui reflètent les sources perçues du produit de terroir, à savoir : le facteur métier, dont les termes savoir-faire, recette et tradition; le facteur temps et culture réunissant les vocables histoire et rituel; et, finalement, le facteur origine rassemblant les mots territoire, région et terre. 
De plus, il ressort que ces trois facteurs n'ont pas le même impact sur les représentations du consommateur liées aux produits de terroir. Ainsi, le facteur métier suscite peu d'associations, tandis que le facteur origine est lié à des associations relatives à la région ou au type de producteur. C'est finalement le facteur temps et culture qui induit le plus grand nombre d'évocations chez le consommateur en relation avec un produit de terroir. S'il s'avérait que les résultats de cette étude exploratoire soient généralisables lors d'une seconde étude plus robuste, cela signifierait qu'il pourrait être judicieux, pour les entreprises agrotouristiques, de miser sur la dimension temps et culture lors de l'adoption d'un positionnement et de l'orientation de la communication marketing intégrée destinée aux touristes ou aux excursionnistes.

De son côté, le rapport du Groupe de travail sur les appellations réservées et les produits du terroir, communément appelé Rapport Desjardins, a été présenté à la ministre de l'Agriculture, des Pêcheries et de l'Alimentation de l'époque, Françoise Gauthier, en octobre 2003. Comme suite à l'étude et afin d'appuyer le développement des régions en tenant compte des efforts des producteurs et des transformateurs qui y vivent, le groupe a proposé les définitions que nous reproduisons au tableau 1.

En outre, le groupe insiste sur la nécessité d'une consultation publique préalable à l'attribution d'une appellation réservée, de

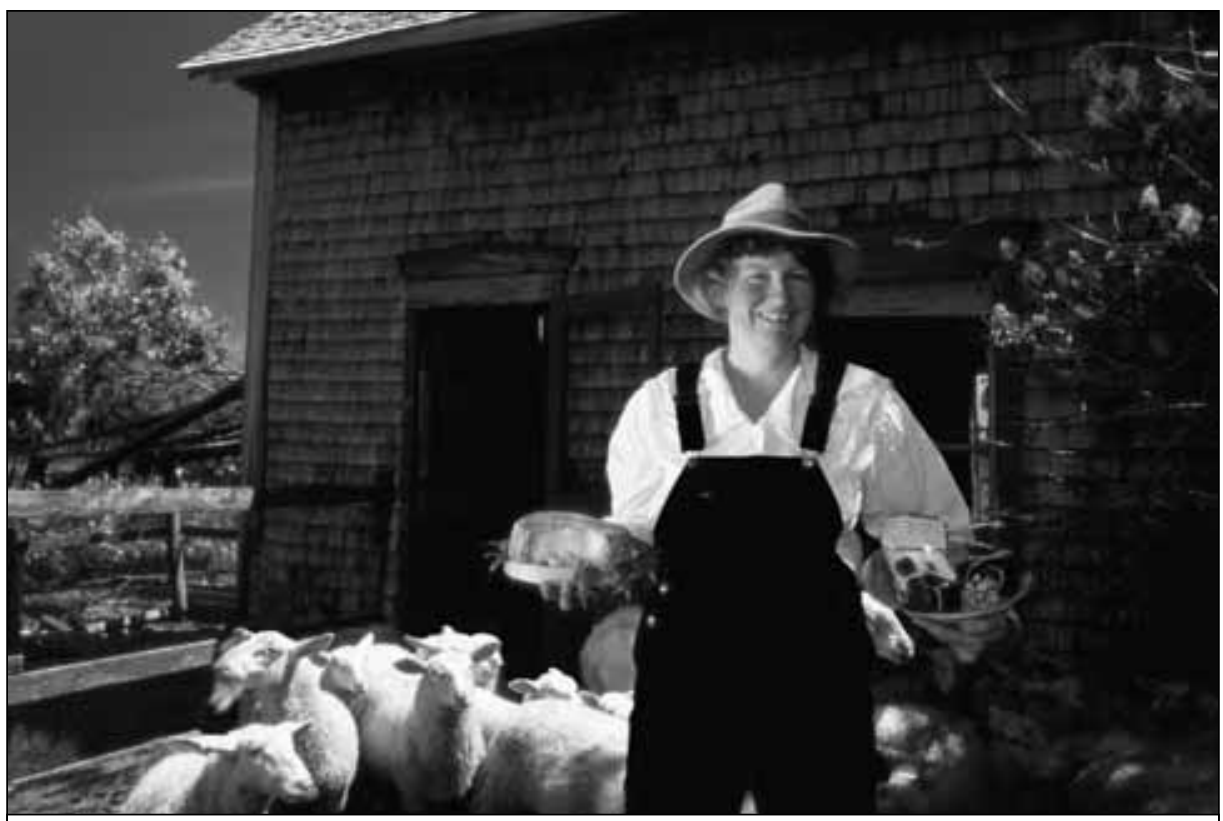

Fromagère et ses produits, Québec.

Photo : @ Tourisme Québec/Ediphoto

même que sur la compatibilité et sur l'harmonisation des appellations avec les normes internationales déjà reconnues, notamment celles en usage dans les pays de l'Union européenne, afin de favoriser l'accès à ces marchés. Par ailleurs, soulignons qu'une harmonisation avec les normes internationales réduirait la confusion relative aux appellations pour les touristes européens et s'intégrerait facilement aux standards de qualité développés par l'industrie touristique.

\section{Tableau 1}

\section{Les définitions des appellations réservées et des produits du terroir}

\begin{tabular}{l|l}
\hline Terme & \multicolumn{1}{c}{ Description } \\
\hline Produit du terroir & $\begin{array}{l}\text { Produit qui provient - ou dont les principales composantes proviennent - d'un ter- } \\
\text { ritoire délimité et homogène et dont les caractéristiques qui le distinguent de façon } \\
\text { significative des produits de même nature reposent sur la spécificité de ce territoire. } \\
\text { Ses caractéristiques dépendent à la fois des particularités du milieu, comme la géo- } \\
\text { logie, le climat, le relief, la culture, l'histoire, ainsi que du savoir et du savoir-faire, } \\
\text { traditionnel ou émergent, de ses habitants. }\end{array}$
\end{tabular}

Produit fermier

Produit artisanal

Produit avec mention de lieu, de territoire ou de région
Produit cultivé, élevé et transformé dans une exploitation agricole, à partir des matières premières qui en proviennent, par son ou ses exploitants, qui gardent le contrôle de sa mise en marché.

Produit résultant d'un mode de production non industrialisé, fabriqué par une personne de métier qui travaille à son compte, aidée ou non par une équipe restreinte, et qui transforme des matières premières, qu'elles soient végétales, animales ou minérales. Notons qu'une distinction s'impose dans le cas des produits alcoolisés.

Produit auquel une mention de lieu, de territoire ou de région est associée et qui doit être issu (produit ou produit et transformé) du lieu, du territoire ou de la région à laquelle se réfère la mention.

\section{Les appellations dans le monde}

L'Union européenne a depuis longtemps mis en place une réglementation communautaire qui régit les appellations d'origine et les indications géographiques protégées dans le domaine de l'agroalimentaire. Une fois adoptées par les autorités en place, ces appellations sont protégées dans tous les états membres. La figure 1 illustre de manière synoptique les diverses appellations et labels de qualité agroalimentaire dans le monde. Essentiellement, trois approches caractérisent le processus d'attribution des appellations. II s'agit notamment des approches territoriale et qualitative, d'une part, et du mode de production, d'autre part.

L'approche territoriale renvoie aux appellations d'origine mettant en valeur les spécificités du terroir qui intègrent les facteurs humains et le savoir-faire de l'homme. À ce titre, I'Indication Géographique Protégée (IGP) signifie l'existence de liens entre le produit et le terroir à au moins un des stades de la production, de la transformation ou de l'élaboration. L'Appellation d'Origine Contrôlée (AOC) indique une dénomination géographique d'une région ou d'un pays et dévoile un trait d'union «produit-terroir », rendant ainsi impossible de reproduire le produit dans un autre terroir. Quant à elle, l'Appellation d'Origine Protégée (AOP) concerne le nom d'un produit pour lequel il existe un savoir-faire reconnu et vérifié et dont la pro-

Source : Rapport Desjardins, 2003 : 10-12. 
Figure 1

Les appellations et les labels de qualité agroalimentaires dans le monde

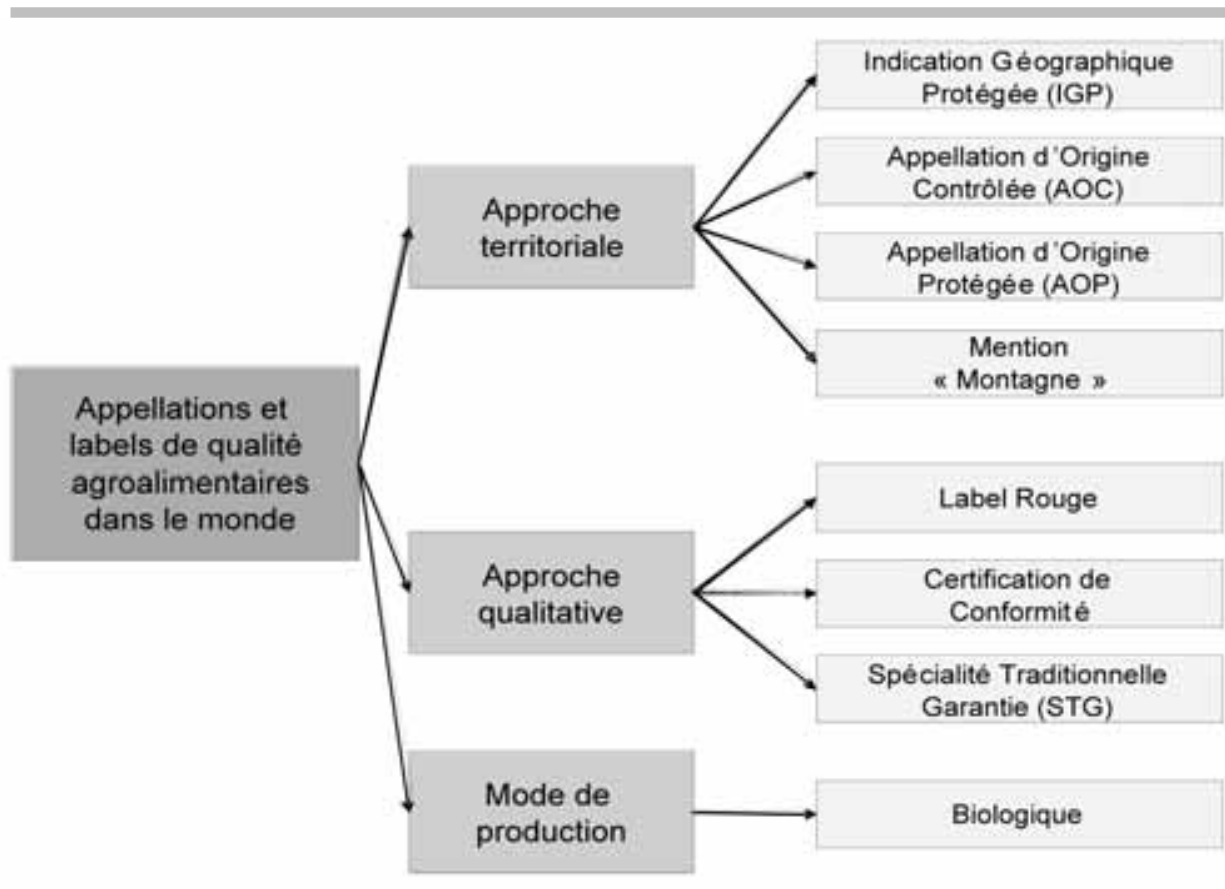

Source : Adapté du Conseil des appellations agroalimentaires du Québec.

duction, la transformation et l'élaboration sont réalisées dans une aire géographique déterminée. Finalement, la désignation Montagne atteste l'origine montagnarde des produits agroalimentaires qui portent cette mention.

L'approche qualitative met un accent moindre sur le terroir et fait plutôt appel à des notions davantage subjectives du produit liées à la qualité, à la tradition, aux particularités de la fabrication déterminantes pour l'attribution de l'appellation. Cette approche repose sur des analyses sensorielles et des tests de dégustation pour analyser des produits confectionnés à partir d'un cahier des charges, qui décrit rigoureusement les procédés de fabrication qui doivent être respectés pour avoir droit à l'appellation convoitée. Ainsi, le Label Rouge certifie que le produit possède un ensemble d'attributs faisant en sorte qu'il offre un niveau de qualité gustative supérieur aux produits courants comparables. II s'agit souvent de produits qui possèdent une renommée mondiale. Par ailleurs, la Certification de Conformité fait référence au fait qu'un produit alimentaire respecte des normes mesurables et préalablement fixées relatives à sa fabrication, à sa transformation et à son conditionnement. En dernier lieu, la désignation Spécialité
Traditionnelle Garantie (STG) souligne la composition ou le mode de production traditionnel du produit, sans toutefois faire référence à son origine.

Finalement, l'approche liée au mode de production dit Biologique réfère à des produits ou à des ingrédients qui proviennent de méthodes de production précises et soumises à un cadre réglementaire strict nécessitant une certification préalable avant d'être mis en marché. Cette appellation est le reflet des préoccupations croissantes de la population quant aux problèmes relatifs à l'exploitation, à la gestion et à la protection de l'environnement agricole. On dénombre actuellement environ 1000 producteurs et transformateurs biologiques officiellement enregistrés auprès des différents organismes de certification. De ce nombre, on recensait près de 902 fermes certifiées biologiques au Québec en 2003 (Fédération d'agriculture biologique du Québec, 2006).

\section{La mise en marché des produits de terroir : un oxymoron?}

Les enjeux liés à la mise en marché des produits de terroir et à leur intégration à l'agrotourisme sont multiples. Bien que le Québec en soit encore à ses balbutiements en cette matière, les exemples d'applications rigoureuses des appellations réservées ont fait école dans plusieurs pays européens. D'abord, il est difficile d'échapper au paradoxe créé par la dynamique du développement mondial et des terroirs. La mondialisation, avec son lot de normes technologiques, de standardisation et d'uniformisation des modes de vie, bénéficie de l'originalité régionale en intégrant des produits et des savoir-faire distinctifs essentiels dans le processus concurrentiel. Or, le succès entraîne une utilisation intensive des matières de base et amène en parallèle la raréfaction de ces ressources «limitées » indispensables pour assurer la différenciation visée par la dynamique concurrentielle. La règle du meilleur incite à rechercher des économies d'échelle qui encouragent le déracinement d'individus dont la typicité des savoir-faire est telle qu'elle ne peut être remplacée dans leur milieu d'origine, entraînant du même coup une diminution de la capacité de ces territoires à perpétuer la différence, source de leur richesse originelle (Solidarité rurale du Québec, 2003). La nécessité d'élaborer une loi sur les appellations réservées et de protéger la production et les acteurs, du secteur des fromages notamment, s'inscrit dans cette logique de vouloir, d'une part, renouveler et diversifier l'économie rurale et, d'autre part, préserver les produits de terroir qui seront partie intégrante d'une stratégie marketing axée sur un agrotourisme authentique.

Les appellations sont indéniablement un enjeu de concurrence internationale qui permet de reconnaître et de valoriser l'économie des terroirs, tout en aidant les territoires visés à se munir d'outils de compétitivité en lien avec la qualité, l'origine et la protection du savoir local. Les appellations permettent aux artisans et aux producteurs fermiers non seulement de différencier leurs produits dans un contexte de concurrence et de segmentation des marchés, mais aussi de favoriser la récupération de la valeur ajoutée associée aux produits dont bénéficieront les habitants locaux et le territoire. C'est le cas rapporté notamment par Bret (2004) pour la région de production du parmigiano-reggiano où les 547 petites fromageries affinent le célèbre fromage produit par les 5842 producteurs.

Le même auteur fait également mention, sous l'égide d'ORIGIN² (Organisation for an International Geographical Indications 
Network), du cas éloquent du fromage comté soulevé lors d'une table ronde organisée à l'occasion d'un symposium de l'Organisation mondiale du commerce (OMC) tenu à Genève en mai 2004. Le comté est un fromage $\mathrm{AOC}$ qui totalise aujourd'hui une production annuelle de 50000 tonnes. Ce nom est protégé par I'AOC comté ; bien que d'autres aient le droit de produire un fromage similaire, ils ne peuvent toutefois utiliser le nom "comté ", à moins que le fromage ait été produit en conformité avec le cahier des charges et dans la zone de production. À l'origine, le massif Jurassien produisait le comté et l'emmental à peu près à parts égales, les technologies de production étant relativement voisines. Or, la production de l'emmental, dont le nom n'a pas été aussi bien protégé, a diminué de 20000 tonnes au cours des 20 dernières années, comparativement à celle du comté qui s'est accrue de 12000 tonnes. Aujourd'hui, 5000 familles d'agriculteurs, 182 petits ateliers de transformation et 20 maisons d'affinage vivent essentiellement du comté. En définitive, l'attribution d'une

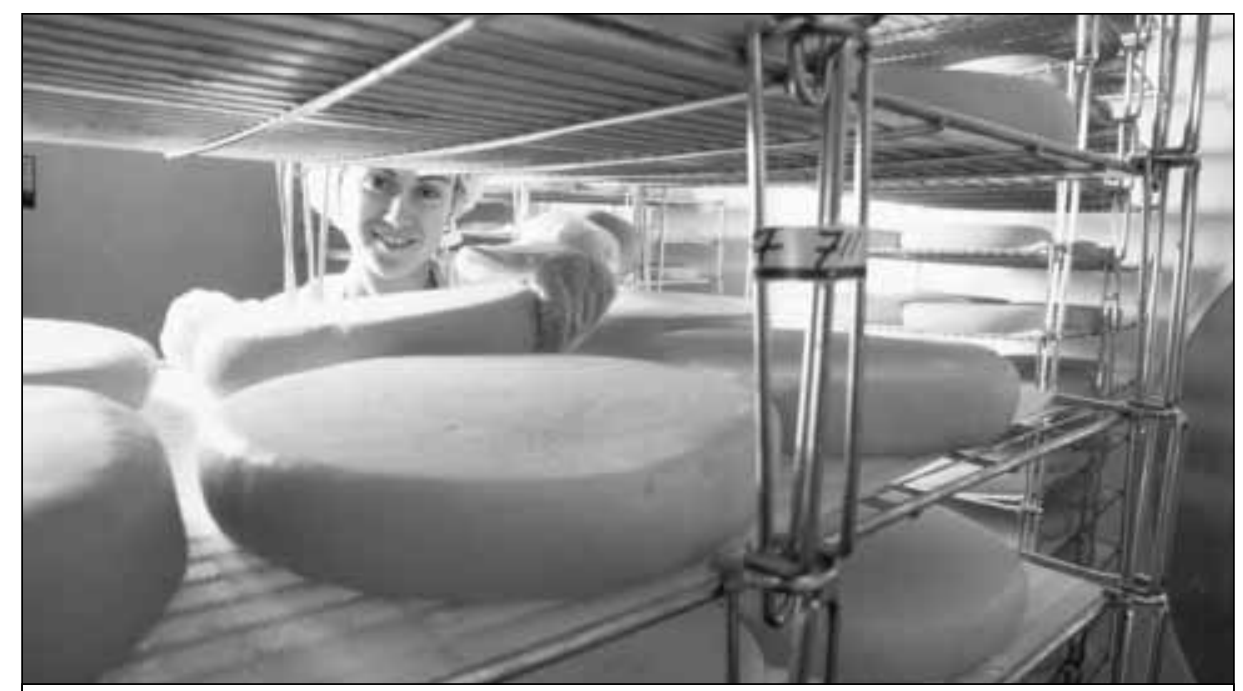

La Maison d'affinage Maurice Dufour à Baie-Saint-Paul. On y fabrique le Migneron, Grand prix 2002 des fromages canadiens.

Photo: ( Tourisme Québec/Marc Archambault
AOC à un produit comme celui-là aura permis de maintenir de nombreux emplois dans une région originellement désavantagée par la nature (Bret, 2004 ; Solidarité rurale du Québec, 2005).

\section{Figure 2}

Impact du terroir sur les relations producteurs, restaurateurs et consommateurs

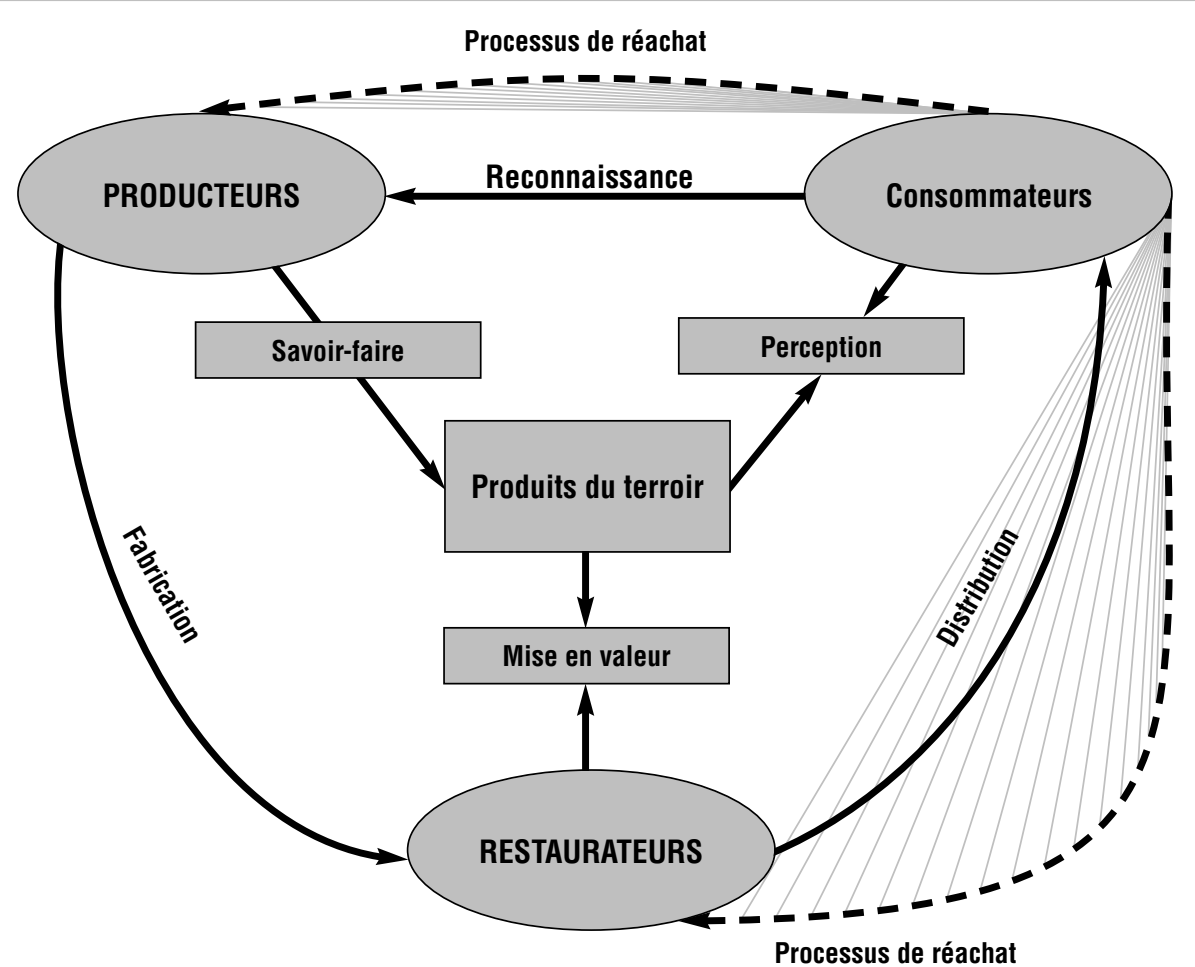

Source : Delatte et Lorrain, $2005: 23$.
On estime qu'il y a 450 sortes de fromages en France (Barcelo, 2003), alors que le Québec en compterait de 180 à 300 (Robillard, 2002). Cet engouement des Québécois pour les fromages fins est à la base de l'intérêt porté par des chercheurs du Laboratoire de recherche et d'intervention en gestion hôtelière et restauration de I'UQTR aux relations existantes entre produits de terroir, producteurs, consommateurs et restaurateurs. Ce sujet a fait l'objet la recherche exploratoire que nous présentons à la prochaine section.

\section{Notre recherche}

Notre recherche exploratoire s'inscrit dans le programme d'activités développé par le Laboratoire de recherche et d'intervention en gestion hôtelière et de restauration de l'UQTR. Cette recherche a été réalisée par cinq étudiants français, encadrés par des professeurs, auteurs de cette étude, lors d'un stage international de formation effectué à l'UQTR à l'été 2005. La figure 2 expose le cadre de référence qui illustre l'articulation des différents concepts utilisés pour cette recherche.

Les acteurs gravitent autour d'une même variable: les produits de terroir. Ces produits possèdent des caractéristiques intrinsèques à la source de la dynamique entre les producteurs, les restaurateurs et les consommateurs-touristes ${ }^{3}$. Par leurs savoir-faire, les producteurs participent à la fabrication de produits de terroir suivant des méthodes artisanales et en respectant divers critères de 
qualité qui caractérisent ces produits. Au cours du processus de distribution qui s'ensuit, les restaurateurs mettent en valeur les caractéristiques distinctives des produits et véhiculent les valeurs que les producteurs y ont préalablement intégrées. Ici la mise en valeur est double puisque, d'une part, la qualité intrinsèque des produits de terroir contribue à l'excellence de la qualité de la cuisine offerte par les restaurateurs aux touristes et aux consommateurs et que, d'autre part, la qualité de la cuisine des restaurateurs met en valeur l'excellence des produits de terroir. Ce mode d'interaction particulier souligne la complémentarité qui existe entre les restaurateurs et le terroir, de même que la création de valeur pour les touristes et les consommateurs lors du processus de transformation.

Les touristes et les consommateurs profitent ainsi du travail combiné des producteurs et des restaurateurs, ce qui les amène à percevoir les attributs particuliers des produits de terroir dont ils font l'expérience. Si cette perception est positive, elle engendre un processus de réachat de type "consommateurs-producteurs » ou "consommateursrestaurateurs ". L'hypothèse que ces deux processus puissent être combinés est aussi envisageable. Ces types de comportements de la part des touristes et des consommateurs contribuent directement à alimenter les conditions de succès du développement de l'offre agrotouristique des entreprises, à savoir la mise en valeur de la production agricole et de ses dérivés, la communication du savoir-faire et une expérience s'intégrant à l'offre touristique des régions du Québec. Du reste, la perception s'établit de manière bilatérale, de sorte que les caractéristiques propres aux produits (savoir-faire, histoire, culture et ancrage géographique) interagissent aussi au moment de l'achat avec le niveau d'expertise, de connaissance ou de familiarité exprimé plus ou moins inconsciemment par les consommateurs au moment de l'achat des produits de terroir. Les touristes et les consommateurs vont ainsi se créer peu à peu une identité alimentaire qu'ils vont essayer de construire au fur et à mesure de leurs "rencontres » avec les deux autres acteurs du circuit.

Notre recherche a été effectuée à partir de trois questionnaires comportant chacun environ 15 questions. Le premier a été administré à 17 producteurs, dont 11 spécialisés dans la fabrication des fromages, le 18 juin 2005, au marché de produits de terroir
Godefroy à Saint-Grégoire près de TroisRivières. Le même jour et au même endroit la version du questionnaire destinée aux consommateurs et aux touristes a été administrée à 130 personnes. Douze chefs de cuisine ont répondu, en face à face sur leur lieu de travail, à la version «restaurateur » du questionnaire (Delatte et Lorrain, 2005). Nous traitons des résultats obtenus en lien avec l'objet de cet article à la section suivante.

\section{Résultats}

Suivant les objectifs de notre recherche, il convient de partager avec le lecteur plusieurs des éléments qui sont ressortis des réponses obtenues. Nous présentons ainsi la compréhension des acteurs interrogés quant à la signification du «terroir », au mode de distribution choisi et aux considérations à l'égard d'un label, de normes ou de certification.

\section{L'allusion au terroir}

D'entrée de jeu, les répondants ont été amenés à fournir les quatre mots qui, selon eux, définissaient le mieux un produit de terroir. Le tableau 2 présente les mots les plus fréquemment utilisés par les répondants.

En comparant les mots utilisés selon les trois points de vue, un produit de terroir se définit comme étant un produit issu de la région, un produit fabriqué de manière artisanale, unique ou authentique, fabriqué en petite quantité, nécessitant un savoir-faire, de même qu'une qualité, un goût et une saveur spécifiques. Notons que seuls les producteurs font référence à la variable historique qu'on trouve dans le mot "tradition». En effet, pour eux, un produit doit s'ancrer dans l'histoire et doit tirer son existence d'une tradition ancestrale. Leur définition rappelle aussi l'aspect technique lié au mot "savoir-faire ", puisque, étant donné que le produit de terroir est fabriqué depuis plusieurs années selon les mêmes techniques ancestrales, les producteurs se doivent de respecter rigoureusement ces méthodes de production, les consommateurs et les touristes y étant très sensibles. Les producteurs sont conscients que le consommateur crée un lien entre le fait que le produit fasse partie du terroir et sa qualité supposée ; pour eux, terroir rime avec qualité, ce qui les incite à acheter davantage. Par ailleurs, les producteurs évoquent la dimension symbolique du mot « artisanal », référant au fait que les produits de terroir sont des produits culturels qui « appartiennent » aux personnes qui habitent la région. Cette mention reflète l'idée suivante: le produit appartient à une région et les gens qui en font partie sont fiers de revendiquer ce produit et n'aiment pas que d'autres se l'approprient. Les réponses fournies par les producteurs nous amènent à penser que, dans l'ensemble, les producteurs ont plutôt une bonne connaissance de ce qu'est un produit de terroir.

Au demeurant, tous les répondants sont massivement convaincus que le Québec possède un terroir distinctif. Certains sont même d'avis que chacune des régions possède un terroir qui lui est propre. Ils n'ont pas hésité à mentionner les catégories de produits qui singularisent le mieux les produits de terroir au Québec. Comme on peut s'y attendre, les produits de l'érable trônent au premier rang, suivis de près par les fromages. Les viandes et les vins sont, dans une moindre mesure, reconnus comme produits de terroir associés au Québec. On trouve également les poissons, les crustacés, la bière, le miel, le chocolat ainsi que les fleurs comestibles dans la liste des produits typiques du Québec mentionnés par les répondants.

\section{Tableau 2}

\begin{tabular}{|c|c|c|c|c|}
\hline \multicolumn{5}{|c|}{ Les quatre mots définissant un produit de terroir } \\
\hline \multirow[t]{2}{*}{ Acteur } & \multicolumn{4}{|c|}{ Ordre de citation } \\
\hline & $1^{\text {er }}$ choix & $2^{\mathrm{e}}$ choix & $3^{\mathrm{e}}$ choix & $4^{e}$ choix \\
\hline Consommateurs/Touristes & Région & Qualité et goût & Biologique & Savoir-faire \\
\hline Restaurateurs & Région & $\begin{array}{l}\text { Qualité } \\
\text { Production limitée }\end{array}$ & $\begin{array}{l}\text { Authenticité } \\
\text { Reconnaissance }\end{array}$ & Savoir-faire \\
\hline Producteurs & Artisanal & Région & Tradition & Savoir-faire \\
\hline
\end{tabular}

Source: Données recueillies par les auteurs. 


\section{Le mode d'approvisionnement choisi}

La fromagerie des producteurs constitue, selon les répondants, le canal de distribution le plus utilisé, à la fois par les producteurs, les consommateurs et les touristes. De leur côté, les consommateurs et les touristes privilégient à peu près à parts égales les épiceries traditionnelles et fines ainsi que le marché pour acheter leurs fromages. Notons qu'environ $36 \%$ des consommateurs et des touristes affirment consommer également des fromages au restaurant à une fréquence de $20 \%$ de leurs visites. Pour leur part, les producteurs utilisent les épiceries fines pour une question d'image et de proximité des clients potentiels. Le réseau des épiceries traditionnelles et des épiceries fines soulage les producteurs de la paperasserie liée à la commande et à la facturation, d'où une économie de temps. Le recours à la fromagerie des producteurs et aux restaurateurs est aussi lié à la gestion de l'image, bien que les restaurateurs engendrent en plus une utilité de proximité. Bref, le choix du circuit de distribution repose sur des considérations qui se rapportent à l'image, à l'aspect économique et à la proximité des clients potentiels. Le tableau 3 met en lumière l'utilisation des canaux de distribution des produits fromagers de terroir par les producteurs et les consommateurs.

\section{Le label, les normes et la certification}

Sur un autre plan, les restaurateurs, les consommateurs et les touristes se sont exprimés sur l'apport éventuel de la mise en

\section{Tableau 3}

Canaux de distribution privilégiés

\begin{tabular}{l|c|c}
$\begin{array}{l}\text { Canal de } \\
\text { distribution }\end{array}$ & Producteur & $\begin{array}{c}\text { Consommateur/ } \\
\text { Touriste }\end{array}$ \\
\hline $\begin{array}{l}\text { Épicerie } \\
\text { traditionnelle } \\
\text { Épicerie fine }\end{array}$ & $13 \%$ & $25 \%$ \\
$\begin{array}{l}\text { Fromagerie } \\
\text { du producteur }\end{array}$ & $25 \%$ & $20 \%$ \\
Marché & $13 \%$ & $28 \%$ \\
Restaurant & $23 \%$ & - \\
Autres(détaillants, & $5 \%$ & $3 \%$ \\
entrepôts) & & \\
\hline
\end{tabular}

Source : Données recueillies par les auteurs.

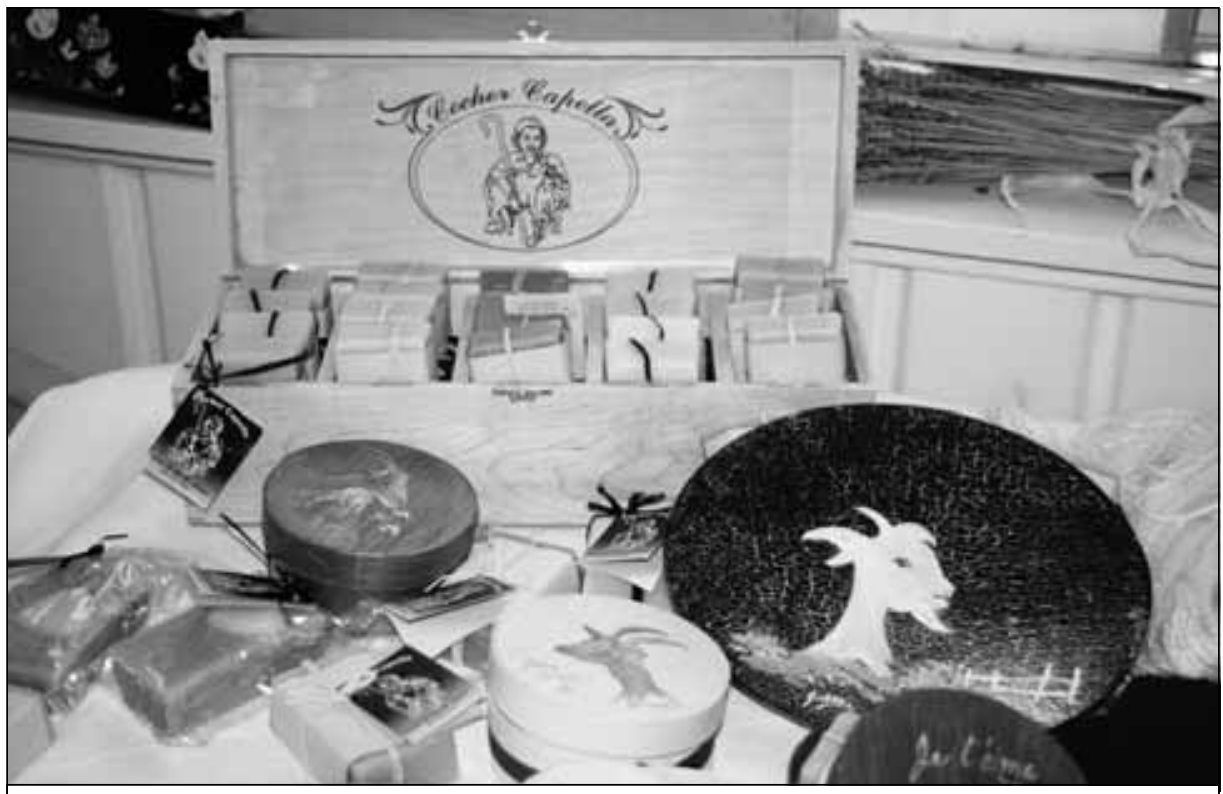

Produits de la chèvre, Cocher Capella, Kingsey Falls.

Photo : @ Tourisme Québec/Michèle Foreman

place d'un label de qualité reconnaissant les produits de terroir. II y a consensus et de loin sur le souhait que le label apporte une assurance d'authenticité des produits de terroir. Pour les restaurateurs, le label conférerait une plus grande reconnaissance et une sécurité accrue quant à l'origine ou au respect du mode de fabrication des produits de terroir. Les consommateurs et les touristes, de leur côté, y voient non seulement une occasion de mieux reconnaître ces produits, mais aussi de les repérer plus facilement, tout en étant assurés que le cahier des charges relatif à leur fabrication soit respecté. L'utilisation d'un label est perçue par les consommateurs et les touristes comme un moyen de préserver ces produits et de souligner leur caractère particulier.

Notons que la mise en place d'un système de certification des appellations est destinée, d'une part, à mettre en valeur les produits de terroir et leurs producteurs et, d'autre part, à sécuriser les consommateurs et les touristes en réduisant la confusion existante à propos des diverses dénominations ou des usurpations éventuelles de dénomination. II ressort que plusieurs acteurs sont disposés à investir leur temps et leur expertise; en revanche, ils s'interrogent sur les diverses interprétations émanant des acteurs qui gravitent autour de ce projet et affirment que l'investissement en argent ou les frais inhérents constitueraient un frein à la mise en œuvre d'un tel système (Trahan, 2005).

\section{Conclusion}

Les résultats fragmentaires que nous avons présentés renforcent notre intime conviction de la nécessité d'entreprendre de nouvelles recherches en ce domaine. La préservation des produits de terroir, dont les fromages, est une étape obligatoire à franchir afin d'assurer le développement de l'agrotourisme. L'authenticité des produits de terroir offerts aux touristes constitue une condition sine qua non du développement d'une offre touristique de qualité. Cet enjeu représente non seulement un impératif sur le plan du commerce international, mais également sur le plan local, pour assurer le développement durable des régions et pour protéger les consommateurs et les touristes des usurpations d'identité que nul ne saurait éviter en l'absence d'une législation à cet effet. Par ailleurs, les résultats de notre recherche exploratoire font ressortir le fait que les consommateurs et les touristes ont une connaissance insuffisante des produits de terroir québécois. Bien qu'une campagne publicitaire destinée aux consommateurs contribue actuellement à mieux faire connaître les fromages fins du Québec, il n'en demeure pas moins qu'il y a encore beaucoup à faire pour que la population soit davantage sensibilisée aux enjeux économiques, sociaux et culturels liés à l'agrotourisme et à la consommation régulière de produits de terroir québécois. 
En terminant, rappelons le rôle primordial des restaurateurs et des chefs dans la stratégie de commercialisation de ces produits et l'importante synergie qui résulte de la collaboration étroite entre les producteurs et les restaurateurs. Précisons aussi que, au moment d'éditer notre article, le ministre de l'Agriculture vient de déposer le projet de loi 137, projet musclé en remplacement du projet de loi 113 déposé en juin dernier et jugé trop laxiste par les acteurs de l'industrie concernés par cette loi. Le projet vise à réprimer toute tentation d'étiquetage trompeur des produits alimentaires dits artisanaux (Presse canadienne, 2005). Un pas dans la bonne direction pour promouvoir le développement de l'agrotourisme!

\section{Léonard Dumas, William Menvielle,} Jocelyn D. Perreault et Denis Pettigrew sont professeurs de marketing au Département des sciences de la gestion de I'Université du Québec à Trois-Rivières. IIs sont membres du Laboratoire de recherche et d'intervention en gestion hôtelière et restauration.

\section{Notes}

1 Les auteurs tiennent à remercier Jean-Pierre Delatte, Anthony Lorrain, Guillemette Salmon, Céline Thomas et Sandra Tsonde pour leur participation à la réalisation de cette étude.

2 Organisme réunissant des producteurs de 24 pays, créé à Genève le 11 juin 2003 afin de protéger et de promouvoir les indications d'origine géographique.

3 Afin de représenter les catégories de touristes, nous nous sommes basés sur la typologie déployée par Ryan (1991) qui précise que le touriste peut être tout à la fois un ambassadeur, un spectateur ou un consommateur. Pour les besoins de notre étude, c'est ce dernier rôle que nous avons convenu de présenter en priorité.

\section{Bibliographie}

Aurier, Philippe, Fatiha Fort, et Lucie Sirieix (2004), "Les produits de terroir du point de vue des consommateurs : sources perçues et associations au terroir ", Actes du XXe Congrès de l'Association française du marketing (AFM), 6-7 mai, St-Malo.

Barcelo, Yan (2003), "Transformation des ressources: La transformation pose plusieurs défis aux régions ", Les Affaires, Montréal, dossier spécial, samedi 15 février, p. 97.
Bérard, Laurence, et Michel Marchesnay (2000), "Le vivant, le culturel et le marchand: Les produits de terroir ", Autrement, Paris, no 194, p. 191-216.

Bret, Jean-Jacques (2004), « Contribution des indications géographiques au développement durable. Exemple du Comté", conférence présentée au Symposium de l'OMC Multilatéralisme à la croisée des chemins, Genève, table ronde de l'Organisation for an International Geographical Indications Network (ORIGIN ) », 24 mai, [http://origin.technomind.be/ fileadmin/origin/PDFs/English/OriGln_in_Action/ OriGln_Events/JJ_Bret.pdf], consulté le 25 novembre 2005.

Camus, S. (2002), «L'authenticité et le processus d'authentification. Pour une étude des perceptions du consommateur ", XVle journées des Instituts d'administration des entreprises (IAE), Paris.

Conseil des appellations agroalimentaires du Québec (CAAQ) (2005), Types d'appellations dans le monde, [http://www.caaq.org/agro alimentaire/appellations/types-appellation.asp], consulté le 20 décembre 2005.

Cova, V., et B. Cova (2001), Alternatives marketing, Dunod, Paris.

Delatte, Jean-Pierre, et Anthony Lorrain (2005), Les produits du terroir québécois, document de recherche non publié. Laboratoire de recherche et d'intervention en gestion hôtelière et restauration, UQTR, Trois-Rivières, juillet.

Delatte, Jean-Pierre, Anthony Lorrain, Guillemette Salmon, Céline Thomas, et Sandra Tsonde (2005), Étude sur les fromages québécois, document de recherche non publié, Laboratoire de recherche et d'intervention en gestion hôtelière et restauration, UQTR, Trois-Rivières, juillet.

Fédération d'agriculture biologique du Québec (2006), Nombre de fermes biologiques au Québec selon leur production principale, [http://www.fabqbio.ca/pages/frames.htm], consulté le 23 janvier 2006.

FSA, Laval (2004), Résultats d'enquête L'agrotourisme permet d'augmenter le chiffre d'affaires et de diversifier les revenus, Faculté des sciences de l'administration, Université Laval, Bulletin août.

Granovetter, Mark (2000), Le marché autrement, Desclée de Brouwers, Paris.

Groupe de travail sur les appellations réservées et les produits du terroir (2003), Rapport Desjardins, présenté à la ministre de l'Agriculture, des Pêcheries et de l'Alimentation, Françoise Gauthier, Agriculture, Pêcheries et Alimentation, Québec, octobre.

Lessard, Laurent (2005), Notes pour l'allocution du ministre de l'Agriculture, des Pêcheries et de l'Alimentation et député de Frontenac, Laurent Lessard, à l'occasion des journées d'audition de la Commission de l'Agriculture, des Pêcheries et de l'Alimentation relativement au projet de loi modifiant la loi sur les appellations réservées (projet de loi $n^{\circ} 113$ ), Québec, Assemblée nationale, 27 septembre.

MAPAQ (2003), Groupe de concertation sur l'agrotourisme au Québec. Ministère de l'Agriculture, des Pêcheries et de l'Alimentation du Québec, [http://www.mapaq.gouv.qc.ca/ NR/rdonlyres/209BB6B6-8B02-40E6-B88F -50CF40D854F2/0/groupe_concertation.pdf], consulté le 27 décembre 2005.

Marchesnay, Michel (2002), « Les PME de terroir : Entre 'GEO' et 'CLIO' stratégies ", dans Actes de la XI conférence de l'Association internationale de management stratégique (AIMS), ESCP-EAP (École supérieure de commerce de Paris - École européenne des affaires), Paris, 5-7 juin.

Marcotte, Pascale, et Laurent Bourdeau (2004), Portraits d'entreprises agrotouristiques au Québec, Groupe de concertation sur l'agrotourisme au Québec, Rapport présenté au ministère de l'Agriculture, des Pêcheries et de I'Alimentation du Québec et à Tourisme Québec.

Presse canadienne (2005), «Produits du terroir : Québec fait le ménage ", La Presse, Montréal, cahier Actualités, 7 décembre.

Radio-Canada (2004), L'Épicerie, document d'archives, reportage du 14 avril, Société RadioCanada, [http://www.radio-canada.ca/ac tualite/lepicerie/docArchives/2004/04/14/re portage2.shtml], consulté le 21 octobre 2005.

Rastoin, Jean-Louis, et Véronique Vissac-Charles (1999), "Le groupe stratégique des entreprises de terroir », Revue Internationale PME, vol. 12, nos 1-2, p 193-200.

Robillard, Alexandre (2002), "Les fromages québécois : P'tit Québec deviendra grand? ", La Presse, Montréal, cahier Actuel, p. B1.

Ryan, C. (1991), Recreationnal Tourism: A Social Science Perspective, Routledge, London.

Solidarité rurale du Québec (2003), La mise en marché des produits du terroir : Défis et stratégies. Solidarité rurale du Québec, Nicolet, Québec.

Solidarité rurale du Québec (2005), Les appellations : Un enjeu de développement durable pour les territoires, Solidarité rurale du Québec, Nicolet, Québec.

Trahan, B. (2005), "Produits du terroir : Distinguer le vrai du faux ", Le Nouvelliste, Trois-Rivières, lundi 21 novembre, section Actualités, p. 3.

Warnier, J.P. (1994), Le paradoxe de la marchandise authentique, imaginaire et consommation de masse, L'Harmattan, Paris. 